\title{
Stochastic versus Deterministic Approach to Coordinated Supply Chain Scheduling
}

\author{
Tadeusz Sawik \\ Department of Operations Research, AGH University of Science and Technology, Al. Mickiewicza 30, 30-059 Kraków, Poland \\ Correspondence should be addressed to Tadeusz Sawik; ghsawik@cyf-kr.edu.pl
}

Received 9 January 2017; Accepted 11 May 2017; Published 19 June 2017

Academic Editor: Anna Pandolfi

Copyright (C) 2017 Tadeusz Sawik. This is an open access article distributed under the Creative Commons Attribution License, which permits unrestricted use, distribution, and reproduction in any medium, provided the original work is properly cited.

\begin{abstract}
The purpose of this paper is to consider coordinated selection of supply portfolio and scheduling of production and distribution in supply chains under regional and local disruption risks. Unlike many papers that assume the all-or-nothing supply disruption pattern, in this paper, only the regional disruptions belong to the all-or-nothing disruption category, while for the local disruptions all disruption levels can be considered. Two biobjective decision-making models, stochastic, based on the wait-and-see approach, and deterministic, based on the expected value approach, are proposed and compared to optimize the trade-off between expected cost and expected service. The main findings indicate that the stochastic programming wait-and-see approach with its ability to handle uncertainty by probabilistic scenarios of disruption events and the much simpler expected value problem, in which the random parameters are replaced by their expected values, lead to similar expected performance of a supply chain under multilevel disruptions. However, the stochastic approach, which accounts for all potential disruption scenarios, leads to a more diversified supply portfolio that will hedge against a variety of scenarios.
\end{abstract}

\section{Introduction}

Unexpected disruptions of material flows have become a major source of concerns in global supply chains over the recent years and coordinated decision-making of supplies, production, and distribution operations under disrupted flows appears to be a crucial issue (e.g., Blackhurst et al. [1] and Hoffmann et al. [2]). While the probability of flow disruptions is very low, their business impact can be huge. For example, flow disruptions in the electronics supply chains due to the great East Japan earthquake of 11 March 2011 and then the catastrophic October flooding in Thailand, where many component manufacturers were concentrated, resulted in huge losses of major electronics producers (e.g., Park et al. [3] and Haraguchi and Lall [4]). Similar losses were experienced by the automotive industry (e.g., Fujimoto and Park [5], Matsuo [6], and Marszewska [7]). For example, two months after the earthquake, Toyota North America, which received up to $15 \%$ of its parts from Japan, experienced a shortage of 150 critical parts and was forced to operate at only $30 \%$ of its capacity.
The business practices of many companies (e.g., Zeng and Xia [8]) provide a typical decision-making environment of supply chains under disruptions. Suppliers are often located in different geographic regions and differ in wholesale prices, delivery lead time, and reliability, while their disruption profiles contain parameters such as disruption probability and fulfillment rate or the percentage of an order that is actually delivered. A popular approach to decision-making in supply chains with disrupted flows is stochastic programming, which is capable of incorporating probabilistic disruption scenarios and finding supply chain coordinated schedules for all potential scenarios with respect to various conflicting objective functions. In this paper, we present an application of stochastic mixed integer programming (stochastic MIP) to coordinated selection of supply portfolio and scheduling of production and distribution in supply chains with partially or fully disrupted supplies. The suppliers are located in different geographic regions and the supplies are subject to partial (multilevel) local disruptions of each supplier individually and to all-or-nothing (two-level) regional disruptions of all suppliers in the same region. Two equally important and 
conflicting objectives are simultaneously optimized: expected cost and expected service level. In this paper, the stochastic MIP approach is compared with a deterministic MIP approach, in which all potential disruption scenarios are replaced by a single scenario, which is obtained by replacing the stochastic parameters by their expected values.

The paper is organized as follows. The review of relevant literature is presented in Section 2. The problem of coordinated decision-making in a supply chain subject to partial local disruptions and all-or-nothing regional disruptions is described in Section 3. The stochastic mixed integer program with the objective of minimizing the weighted sum of expected cost and expected service level is developed in Section 4 and the corresponding deterministic mixed integer program is proposed in Section 5. Numerical examples, computational results, and some comparison of the two solution approaches are provided in Section 6. Finally, conclusions and managerial implications as well as directions for further research are presented in Section 7.

\section{Literature Review}

The literature on coordinated decision-making in production-distribution planning and scheduling is mostly limited to deterministic models with the supply operation considered separately (e.g., Erengüç et al. [9]). For example, an integrated production, inventory, and distribution routing problem and a MIP approach combined with a heuristic routing algorithm to coordinate the production, inventory, and transportation operations was considered by Lei et al. [10]. Kaur et al. [11] proposed a graph theoretic approach for supply chain coordination to model various mechanisms of coordination and their interdependencies. A digraph representing the supply chain coordination is converted to its adjacency matrix whose permanent function gives a composite index of coordination. In Choi et al. [12], a single- or multisupplier and single-manufacturer supply chain scheduling and coordination problem was formulated as a twomachine common-due-window flow shop scheduling problem. The authors developed scheduling algorithms for both the decentralized (with the manufacturer as a decisionmaker) and centralized supply chains. Liu and Papageorgiou [13] developed a multiobjective MIP approach to address production, distribution, and capacity planning of global supply chains considering cost, responsiveness, and service level simultaneously. Chen [14] presented a review of existing models that integrate production and outbound distribution operations at the detailed scheduling level. The models aim at optimizing detailed order-by-order production and delivery scheduling jointly by taking into account relevant revenues, costs, and service levels at the individual order level. Sawik [15] proposed a MIP approach for integrated scheduling of material manufacturing, material supply, and product assembly in a customer driven supply chain. A monolithic approach, where the manufacturing, supply, and assembly schedules are determined simultaneously, was compared with a hierarchical approach. Numerical examples modeled after real-world scheduling in the electronics supply chain were reported.
Mitigation and contingency/recovery actions were studied by Tomlin [16] in a dual sourcing setting, one unreliable supplier and another reliable and more expensive one. A buyer that suffers a supply shortage can buy from a more expensive alternate supplier or produce less and its decision depends on its inventory. If the reliable supplier has volume flexibility, contingent rerouting by temporarily increasing its production may prove to be an effective way to speed up recovery process. The author established that, along with cost, percentage of supplier uptime, disruption length, capacity, and flexibility play an important role in determining a buyer's disruption management strategy. The recent developments in the field of supply chain disruption management from a multidisciplinary perspective were summarized by Ivanov et al. [17-19] who studied the Ripple effect in supply chains. They emphasized that the Ripple effect can consolidate research in supply chain disruption management, similar to the bullwhip effect regarding demand and lead time fluctuations.

In the literature on supply uncertainty, the supply is subject to either complete disruptions or yield uncertainty. Yield uncertainty occurs when the quantity of supply delivered is a random variable, modeled as either a random additive or multiplicative quantity, whereas disruptions occur when supply is subject to partial or complete failure. Typically disruptions are modeled as events which occur randomly and may have a random length. Schmitt and Snyder [20] considered inventory systems subject to both supply disruptions and yield uncertainty. They compared single-period versus multiperiod models and showed that the former can lead to selecting the wrong strategy for mitigating supply risk. Schmitt et al. [21] investigated optimal system design in a multilocation system under supply disruptions. They examined the expected costs and cost variances of the system in both centralized and decentralized inventory systems. They showed that when demand is deterministic and supply is disrupted, a decentralized inventory reduces cost variance through the risk diversification effect and that a decentralized inventory may also be selected when supply is disrupted and demand is stochastic. A recent literature review on OR/MS models for supply chain disruptions was presented by Snyder et al. [22]. They discussed 180 scholarly works on the topic, organized into six categories: evaluating supply disruptions, strategic decisions, sourcing decisions, contracts and incentives, inventory, and facility location.

Sawik [23, 24] proposed a new stochastic MIP approach to integrated selection of supply portfolio and scheduling of customer orders in a supply chain under all-or-nothing disruption risks. The stochastic MIP formulations were further enhanced by Sawik [25] to jointly optimize supply portfolio and production and distribution of finished products. For the distribution of products, three shipping methods were considered and compared.

This paper differs from the previous research in the following two aspects. First, unlike many articles that assume the all-or-nothing supply disruption pattern, in this paper, only the regional disruptions belong to the all-or-nothing disruption category. For the local disruptions, however, all disruption levels can be considered within three categories: minor disruption, major disruption, and complete shutdown 
(e.g., $[8,26])$. Disruption profiles contain parameters such as probability of disruption at all levels and fulfillment rate or the percentage of an order that is actually delivered. Second, in this paper, a stochastic programming wait-andsee approach with its ability to handle uncertainty by probabilistic scenarios of disruption events is compared with a deterministic programming approach, in which the random parameters are replaced by their corresponding expected values to achieve the so-called expected value problem (e.g., Kall and Mayer [27]). The expected value problem is a MIP and is often used in practice as the related stochastic mixed integer program is in general much harder to solve, since it considers multiple scenarios (e.g., Durbach and Stewart [28] and Maggioni and Wallace [29]). The objective of both the wait-and-see approach and the expected value approach is to optimize expected performance of a supply chain under the two types of disruptions with respect to two conflicting objective functions, expected cost and expected service. While the stochastic approach aims at optimizing the expected performance of a supply chain over all possible disruption scenarios, the deterministic approach accounts only for a single scenario representing the expected disruption conditions. The stochastic programming approach determines a subset of nondominated solutions for all disruption scenarios, whereas the deterministic approach produces a nondominated solution for a single scenario only. The solution of the expected value problem does not take into account any distribution information and remains the same as long as the expectations do not change. Unlike the expected value problem, stochastic programming provides a recommendation for selection of supply portfolio that will hedge against a variety of disruption scenarios. The two approaches and the corresponding solutions are compared and some managerial insights derived.

\section{Problem Description}

Consider a three-echelon supply chain (see Figure 1), in which a single producer of one product type assembles and delivers products to multiple distribution centers to meet customer demand, using a critical part type that can be manufactured and provided by many suppliers.

Let $I=\{1, \ldots, \bar{I}\}$ be the set of $\bar{I}$ suppliers, let $J=\{1, \ldots, \bar{J}\}$ be the set of $\bar{J}$ customers, let $K=\{1, \ldots, \bar{K}\}$ be the set of $\bar{K}$ distribution centers, and let $T=\{1, \ldots, \bar{T}\}$ be the set of $\bar{T}$ planning periods (for notations, see Notations).

The orders for parts are assumed to be placed at the beginning of the planning horizon, and the parts ordered from supplier $i$ are delivered in period $\sigma_{i}$. Each customer is supplied with the ordered products via exactly one distribution center. The products for each customer $j \in J_{k}$ are delivered to the distribution center $k$ in a single delivery, which cannot be scheduled before all customer orders $j \in J_{k}$ have been completed. The products shipped in period $t$ to distribution center $k$ are delivered in period $t+\tau_{k}-1$.

The suppliers of parts are located in $\bar{R}$ geographic regions. The supplies are subject to random local disruptions of different levels, $l \in L=\{0, \ldots, \bar{L}\}$, where the disruption level refers to the fraction of an order that can be delivered (fulfillment rate). Level $l=0$ represents complete shutdown of a supplier, that is, no order delivery, while level $l=\bar{L}$ represents normal conditions with no disruption, that is, full order delivery. The intermediate disruption levels $l=1, \ldots, \bar{L}-1$ represent different fractions of an order that can be delivered. The smaller $l \in L$, the smaller portion of an order that can be delivered due to the smaller fraction of the supplier capacity available. The fraction of an order that can be delivered by supplier $i$ under disruption level $l$ is described by the associated fulfillment rate, $\gamma_{i l}$ :

$$
\gamma_{i l}= \begin{cases}0, & \text { if } l=0, \\ \in(0,1), & \text { if } l=1, \ldots, \bar{L}-1, \\ 1, & \text { if } l=\bar{L} .\end{cases}
$$

Denote by $p_{i l}$ the probability of disruption level $l \in L$ for supplier $i$; that is, the parts ordered from supplier $i$ are delivered fully with probability $p_{i \bar{L}}$, partially at different levels of supplier output, $\gamma_{i l}$, with probability $p_{i l}, l=1, \ldots, \bar{L}-1$, or not at all with probability $p_{i 0}$.

In addition to independent local disruptions of each supplier, there are potential regional disasters that may result in complete shutdown of all suppliers in the same region simultaneously. For example, regional disaster events may include an earthquake and flooding. Let $p^{r}$ be the probability of regional disruptions of all suppliers $i \in I^{r}$ in region $r \in R$.

Denote by $S=\{1, \ldots, \bar{S}\}$ the index set of all disruption scenarios, where each scenario $s \in S$ can be represented by an integer-valued vector $\lambda_{s}=\left(\lambda_{1 s}, \ldots, \lambda_{\overline{I s}}\right)$, where $\lambda_{i s} \in L$ is the disruption level of an order delivery from supplier $i \in I$ under scenario $s \in S$. All potential disruption scenarios will be considered; that is, $\bar{S}=(\bar{L}+1)^{\bar{I}}$.

The probability $P_{s}$ for disruption scenario $s \in S$ with the subset $I_{s}$ of nonshutdown suppliers (that can deliver parts under scenario $s$ ) is [26]

$$
P_{s}=\prod_{r \in R} P_{s}^{r},
$$

where $P_{s}^{r}$ is the probability of realizing of disruption scenario $s$ for suppliers in $I^{r}$ :

$$
P_{s}^{r}= \begin{cases}\left(1-p^{r}\right) \prod_{i \in I^{r}}\left(p_{i, \lambda_{i s}}\right), & \text { if } I^{r} \cap I_{s} \neq \emptyset, \\ p^{r}+\left(1-p^{r}\right) \prod_{i \in I^{r}} p_{i 0}, & \text { if } I^{r} \cap I_{s}=\emptyset\end{cases}
$$

and $p_{i, \lambda_{i s}}$ is the probability of occurrence of the disruption at level $l=\lambda_{i s}$ of an order delivery from supplier $i$ under scenario $s$.

The objective of the coordinated decision-making in a supply chain under multilevel disruptions is to allocate the total demand for parts among a subset of selected suppliers (i.e., to determine the supply portfolio) and to schedule for each disruption scenario the customer orders for products and the delivery of products to distribution centers to optimize the trade-off between expected cost and expected service level. 


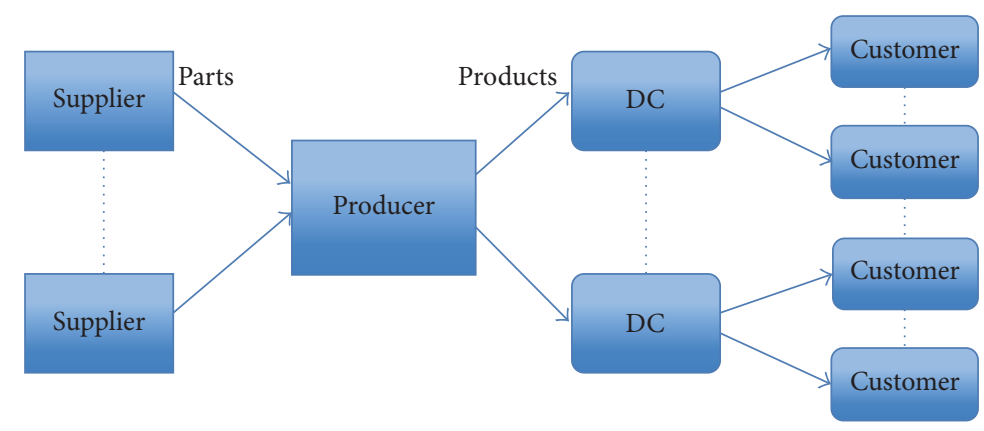

FIgURE 1: A three-echelon supply chain.

\section{Problem Formulation: Stochastic Approach}

In this section, a stochastic MIP model WCS is presented for the coordinated decision-making in the presence of supply chain under multilevel disruption risks. The following decisions are jointly made using the proposed model $[25,26]$ :

(i) Supply portfolio selection: $u_{i}=1$, if supplier $i$ is selected; otherwise $u_{i}=0$, and $v_{i} \in[0,1]$, the fraction of total demand for parts ordered from supplier $i$

(ii) Production scheduling: $w_{j t}^{s}=1$, if under disruption scenario $s$ customer order $j$ is scheduled for period $t$; otherwise $w_{j t}^{s}=0$ (iii) Distribution scheduling: $x_{k t}^{s}=1$, if under disruption scenario $s$ a shipment of products to distribution center $k$ is scheduled for period $t$; otherwise $x_{k t}^{s}=0$

(iv) Customer order nondelayed delivery: $y_{j}^{s}=1$, if under disruption scenario $s$ customer order $j$ is delivered by its due date; otherwise $y_{j}^{s}=0$

The demand allocation vector $\left(v_{1}, \ldots, v_{\bar{I}}\right)$, where $\sum_{i \in I} v_{i}=$ 1 and $0 \leq v_{i} \leq 1, i \in I$, defines the supply portfolio, introduced by Sawik [30].

Let $E_{1}$ be the minimized expected cost per product and let $E_{2}$ be the maximized expected service level:

$$
\begin{aligned}
& E_{1}=\frac{\sum_{i \in I} e_{i} u_{i}+\sum_{s \in S} P_{s}\left(\sum_{i \in I_{s}} B o_{i} \gamma_{i, \lambda_{i s}} v_{i}+\sum_{j \in J} g_{j} b_{j}\left(\sum_{t \in T} w_{j t}^{s}-y_{j}^{s}\right)+\sum_{j \in J} h_{j} b_{j}\left(1-\sum_{t \in T} w_{j t}^{s}\right)\right)}{B}, \\
& E_{2}=\frac{\sum_{j \in J} \sum_{s \in S} P_{s} b_{j} y_{j}^{s}}{B},
\end{aligned}
$$

where $\lambda_{i s}$ is disruption level of supplier $i$ under scenario $s$ and $\gamma_{i, \lambda_{i s}}$ is the corresponding fulfillment rate, that is, the fraction of an order delivered by supplier $i$ under disruption scenario $s$.

The expected cost $E_{1}$ (see (4)) constitutes fixed ordering cost, $\sum_{i \in I} e_{i} u_{i}$, expected purchasing cost for delivered parts, $\sum_{s \in S} P_{s} \sum_{i \in I_{s}} B o_{i} \gamma_{i, \lambda_{i s}} v_{i}$, expected penalty for delayed customer demand, $\sum_{s \in S} P_{s} \sum_{j \in J} g_{j} b_{j}\left(\sum_{t \in T} w_{j t}^{s}-y_{j}^{s}\right)$, and expected penalty for unsatisfied (rejected) customer demand, $\sum_{s \in S} P_{s} \sum_{j \in J} h_{j} b_{j}\left(1-\sum_{t \in T} w_{j t}^{s}\right)$.

Denote by

$$
f_{1}=\frac{E_{1}-\underline{E}_{1}}{\bar{E}_{1}-\underline{E}_{1}}
$$

the normalized (scaled into the interval $[0,1])$ expected cost per product $\left(\underline{E}_{1}, \bar{E}_{1}\right.$ are the minimum and the maximum values of $E_{1}$, resp.) and by

$$
f_{2}=\frac{\bar{E}_{2}-E_{2}}{\bar{E}_{2}-\underline{E}_{2}}
$$

the normalized expected service level $\left(\underline{E}_{2}, \bar{E}_{2}\right.$ are the minimum and the maximum values of $E_{2}$, resp.).

Model WCS. It consists in supplier selection, customer order, and distribution scheduling to minimize Weighted sum of normalized expected Cost and expected Service level.

Minimize

$$
\alpha f_{1}+(1-\alpha) f_{2}
$$

where $0 \leq \alpha \leq 1$, subject to (4)-(7).

\section{Supply Portfolio Selection Constraints}

(i) The total demand for parts must be fully allocated among the selected suppliers.

(ii) Demand for parts cannot be assigned to nonselected suppliers:

$$
\sum_{i \in I} v_{i}=1 \quad v_{i} \leq u_{i} ; i \in I
$$




\section{Customer Order Scheduling Constraints}

(i) For each disruption scenario $s$, each customer order $j$ is either scheduled during the planning horizon $\left(\sum_{t \in T} w_{j t}^{s}=1\right)$ or unscheduled and rejected $\left(\sum_{t \in T} w_{j t}^{s}=0\right)$.

(ii) For any period $t$ and each disruption scenario $s$, the total demand on capacity of all customer orders scheduled in period $t$ must not exceed the producer capacity:

$$
\begin{gathered}
\sum_{t \in T} w_{j t}^{s} \leq 1 ; \quad j \in J, s \in S, \\
\sum_{j \in J} b_{j} w_{j t}^{s} \leq C ; \quad t \in T, s \in S .
\end{gathered}
$$

\section{Supply-Production-Distribution Coordinating Constraints}

(i) For each disruption scenario $s$ and each planning period $t$, the cumulative demand for parts of all customer orders scheduled in period 1 through $t$ cannot exceed the cumulative deliveries of parts in period 1 through $t-1$, from the nonshutdown suppliers $i \in I_{s}$.

(ii) For each disruption scenario, shipment to distribution center $k$ can be scheduled only after the latest completion period of scheduled customer orders $j \in$ $J_{k}$ :

$$
\begin{aligned}
\sum_{j \in J} \sum_{t^{\prime} \in T: t^{\prime} \leq t} b_{j} w_{j t^{\prime}}^{s} & \leq B \sum_{i \in I_{s}: \sigma_{i} \leq t-1} \gamma_{i, \lambda_{i s}} v_{i} ; \quad t \in T, s \in S, \\
\sum_{t \in T K} t x_{k t}^{s} & \geq \sum_{t \in T}(t+1) w_{j t}^{s} ;
\end{aligned}
$$

$$
k \in K, j \in J_{k}, s \in S \text {, }
$$

where $T K=\left\{\min _{i \in I} \sigma_{i}+2, \ldots, \bar{T}+1\right\}$ is the set of shipping periods.

\section{Shipping Constraints}

(i) For each disruption scenario, at most one shipment can be scheduled to each distribution center:

$$
\sum_{t \in T K} x_{k t}^{s} \leq 1 ; \quad k \in K, s \in S .
$$

\section{Customer Due Date Meeting Constraints}

(i) For each disruption scenario $s \in S$, customer order $j \in J_{k}$ can be delivered without delay (i.e., $y_{j}^{s}=$ 1 ), if it is scheduled not later than $d_{j}-\tau_{k}$ and shipped to distribution center $k$ not later than $d_{j}-$ $\tau_{k}+1$; otherwise the customer order is delayed or unscheduled (i.e., $y_{j}^{s}=0$ ):

$$
\begin{aligned}
& y_{j}^{s} \leq \sum_{t \in T: t \leq d_{j}-\tau_{k}} w_{j t}^{s} ; \\
& k \in K, \quad j \in J_{k}, s \in S,
\end{aligned}
$$

$$
\begin{array}{r}
y_{j}^{s} \leq \sum_{t \in T K: t \leq d_{j}-\tau_{k}+1} x_{k t}^{s} ; \\
k \in K, j \in J_{k}, s \in S, \\
\sum_{t \in T: t \leq d_{j}-\tau_{k}} w_{j t}^{s}+\sum_{t \in T K: t \leq d_{j}-\tau_{k}+1} x_{k t}^{s}-1 \leq y_{j}^{s} ; \\
k \in K, j \in J_{k}, s \in S .
\end{array}
$$

Nonnegativity and Integrality Conditions

$$
\begin{array}{cl}
u_{i} \in\{0,1\} ; \quad & i \in I, \\
v_{i} \in[0,1] ; & i \in I, \\
w_{j t}^{s} \in\{0,1\} ; \quad j \in J, t \in T, s \in S, \\
x_{k t}^{s} \in\{0,1\} ; \quad k \in K, t \in T K, s \in S, \\
y_{j}^{s} \geq 0 ; \quad j \in J, \quad s \in S .
\end{array}
$$

Note that $y_{j}^{s}$ does not need to be restricted to being binary, since, for any feasible solution satisfying constraints (14), $y_{j}^{s}$ is always binary.

Model WCS is a deterministic equivalent mixed integer program of stochastic mixed integer program. When this problem is solved, a recommendation is obtained for selection of supply portfolio $\left(u_{i}, v_{i}\right)$ that will hedge against a variety of disruption scenarios in which fulfillment rates of certain suppliers are not sufficient to satisfy demand for parts. The scheduling variables $w_{j t}^{s}, x_{k t}^{s}$ (and $y_{j}^{s}$ ) are decisions that will be implemented in the future, when scenario $s \in S$ is finally realized.

Model WCS illustrates the wait-and-see approach (e.g., [27]). Basically, this approach is based on perfect information about the future. Model WCS can be decomposed into a two-stage stochastic mixed integer program with recourse. The supply portfolio selection variables $\left(u_{i}, v_{i}\right)$ are referred to as first-stage decisions, and the scheduling variables $\left(w_{j t}^{s}, x_{k t}^{s}, y_{j}^{s}\right)$ are referred to as recourse or second-stage decisions. Unlike the first-stage decisions, the latter variables are dependent on the scenario $s \in S$.

Stochastic mixed integer programs are usually hard to solve because they are large-scale optimization problems when applied to real-world problems. A common approach (e.g., [27]) is to consider a simpler deterministic program, known as expected value problem, in which the random parameters are replaced by their expected values or to consider several deterministic programs, each of which corresponds to one particular scenario, and then to combine the obtained solutions into a single heuristic solution.

\section{Problem Formulation: Deterministic Approach}

In this section, the expected value problem EWCS is presented for the coordinated supply chain scheduling under 
expected supply conditions. In model WCS, where the randomness is characterized by a set of disruption scenarios, the only random parameters are suppliers fulfillment rates, $\gamma_{i, \lambda_{i s}}$, which appear both in the objective function (4) and in constraints (11).

In model EWCS, suppliers probabilistic fulfillment rates defined for each disruption scenario, $\gamma_{i, \lambda_{i s}}, i \in I, s \in S$, or equivalently for each disruption level, $\gamma_{i l}, i \in I, l \in L$, (1) have been replaced by the expected fulfillment rates of each supplier:

$$
\Gamma_{i}=\sum_{s \in S} P_{s} \gamma_{i, \lambda_{i s}} ; \quad i \in I
$$

or equivalently

$$
\Gamma_{i}=\left(1-p^{r}\right) \sum_{l \in L} p_{i l} \gamma_{i l} ; \quad i \in I^{r}, r \in R
$$

Accordingly, stochastic binary decision variables, $w_{j t}^{s}, x_{k t}^{s}, y_{j}^{s}$, (17)-(19), defined for each disruption scenario $s \in S$ have been replaced by their deterministic equivalents $W_{j t}, X_{k t}$, $Y_{j}$.

Now, the expected cost per product, $E_{1}$ (see (22)), and the expected service level, $E_{2}$ (see (23)), are defined as follows:

$$
\begin{aligned}
& E_{1}=\frac{\sum_{i \in I} e_{i} u_{i}+\sum_{i \in I} B o_{i} \Gamma_{i} v_{i}+\sum_{j \in J} g_{j} b_{j}\left(\sum_{t \in T} W_{j t}-Y_{j}\right)+\sum_{j \in J} h_{j} b_{j}\left(1-\sum_{t \in T} W_{j t}\right)}{B}, \\
& E_{2}=\frac{\sum_{j \in J} b_{j} Y_{j}}{B} .
\end{aligned}
$$

Model EWCS is presented below.

\section{Model EWCS}

Minimize (8)

subject to

(6), (7), (9), (22), (23),

$\sum_{t \in T} W_{j t} \leq 1 ; \quad j \in J$,

$\sum_{j \in J} b_{j} W_{j t} \leq C ; \quad t \in T$,

$\sum_{j \in J} \sum_{t^{\prime} \in T: t^{\prime} \leq t} b_{j} W_{j t^{\prime}} \leq B \sum_{i \in I: \sigma_{i} \leq t-1} \Gamma_{i} v_{i} ; \quad t \in T$,

$\sum_{t \in T K} t X_{k t} \geq \sum_{t \in T}(t+1) W_{j t}$

$k \in K, \quad j \in J_{k}$,

$\sum_{t \in T K} X_{k t} \leq 1 ; \quad k \in K$,

$Y_{j} \leq \sum_{t \in T: t \leq d_{j}-\tau_{k}} W_{j t} ; \quad k \in K, j \in J_{k}$,

$Y_{j} \leq \sum_{t \in T K: t \leq d_{j}-\tau_{k}+1} X_{k t} ; \quad k \in K, j \in J_{k}$,

$\sum_{t \in T: t \leq d_{j}-\tau_{k}} W_{j t}+\sum_{t \in T K: t \leq d_{j}-\tau_{k}+1} X_{k t}-1$

$\leq Y_{j} ; \quad k \in K, j \in J_{k}$,

$u_{i} \in\{0,1\} ; \quad i \in I$,

$v_{i} \in[0,1] ; \quad i \in I$

$$
\begin{aligned}
& W_{j t} \in\{0,1\} ; \quad j \in J, t \in T, \\
& X_{k t} \in\{0,1\} ; \quad k \in K, t \in T K, \\
& Y_{j} \geq 0 ; \quad j \in J .
\end{aligned}
$$

Notice that, unlike the stochastic programming model WCS which is formulated to determine optimal schedules for all potential disruption scenarios, model EWCS accounts for a single scenario only, representing the expected supplies. Except for the expected values of the random parameters, this model does not take into account any distribution information and the solution remains the same as long as the expectations do not change. In contrast to model WCS, where the selection of supply portfolio, $\left(v_{1}, \ldots, v_{\bar{I}}\right)$, is combined with supply chain scheduling for all disruption scenarios considered, now the portfolio is determined along with a single schedule. [27]

If random parameters appear only in the constraints, then

$$
\mathrm{EV} \leq \mathrm{WS}
$$

where EV is the optimal solution value of the expected value problem EWCS and WS is the optimal solution value of the wait-and-see problem WCS. On the other hand, when uncertainty is limited to the objective function of the problem, the solution obtained by simply replacing the random parameters with their expected values provides already a robust alternative (Delage et al. [31]).

\section{Computational Examples}

In this section, some computational examples are presented to illustrate possible applications of the proposed MIP models and to compare the wait-and-see and the expected value 
approaches. The examples are modeled in part after a realworld electronics supply chain (e.g., Sawik, [32]). The following parameters have been selected for the computational examples.

$\bar{I}=4$ suppliers, $\bar{J}=20$ customer orders, $\bar{K}=2$ distribution centers, $\bar{L}=3$ partial disruption levels, $\bar{R}=2$ geographic regions, and $\bar{T}=7$ planning periods.

$I^{1}=\{1,2\}, I^{2}=\{3,4\}$.

$J_{1}=\{1, \ldots, 10\}, J_{2}=\{11, \ldots, 20\}$.

Shipping times from suppliers: $\sigma=(1,1,3,3)$.

Shipping times to distribution centers: $\tau=(1,2)$.

Customer demand: $b_{j} \in\{2000,3000, \ldots, 10000\}$, for all $j \in J$, and total demand: $B=100000$.

Due dates: $d_{j} \in\left\{2+\min _{i \in I}\left(\sigma_{i}\right)+\min _{k \in K}\left(\tau_{k}\right), \ldots, \bar{T}+\right.$ $\left.\max _{k \in K}\left(\tau_{k}\right)\right\}$ for all $j \in J$. 13000).

Fixed ordering costs for suppliers: $e=(8000,6000,12000$,

Unit purchasing prices from suppliers: $o=(14,12,8,9)$.

Unit penalties for delayed, unfulfilled customer orders: $g_{j}=\left\lceil\max _{i \in I} o_{i} / 350\right\rceil,=1, h_{j}=2 \max _{i \in I} o_{i}=28$, respectively, for all $j \in J$.

Producer capacity: $C=45000$.

Local disruption levels and the associated fulfillment rates (the percentage of an order that can be delivered) are shown below.

$L=\{0,1,2,3\}$, where $l=0$, complete shutdown, $\gamma_{i 0}=0 \forall i \in I$, that is, $0 \%$ of an order delivered; $l=1$, major disruption, $\gamma_{i 1} \in[0.01,0.50] \forall i \in I^{1}$ and $\gamma_{i 1} \in$ $[0.01,0.30] \forall i \in I^{2}$, that is, $1 \%$ to $50 \%$ and $1 \%$ to $30 \%$ of an order delivered, respectively; $l=2$, minor disruption, $\gamma_{i 2} \in[0,51,0.99] \forall i \in I^{1}$ and $\gamma_{i 2} \in[0,31,0.99] \forall i \in I^{2}$, that is, $51 \%$ to $99 \%$ and $31 \%$ to $99 \%$ of an order delivered, respectively; $l=\bar{L}=3$, no disruption, $\gamma_{i 3}=1 \forall i \in I$, that is, $100 \%$ of an order delivered.

The total number of all potential scenarios is $\bar{S}=(\bar{L}+$ $1)^{\bar{I}}=4^{4}=256$ scenarios, where each scenario $s \in S$ is represented by vector $\lambda_{s}=\left(\lambda_{1 s}, \ldots, \lambda_{4 s}\right)$, where $\lambda_{i s} \in L, i \in I$ (see Table 1).

The local nondisruption probability (level $l=3$ ), $p_{i 3}$, was uniformly distributed over $[0.89,0.99]$ and $[0.79,0.89]$, respectively, for suppliers $i \in I^{1}$ and $i \in I^{2}$; that is, the probabilities were drawn independently from $U[0.89 ; 0.99]$ and $U[0.79 ; 0.89]$, respectively.

Given local nondisruption probabilities, $p_{i 3}, i \in I$, the probabilities for the remaining local disruption levels $l=$ $0,1,2$ were calculated as follows:

Probability of complete shutdown (level $l=0): p_{i 0}=$ $0.1\left(1-p_{i 3}\right)$

Probability of major disruption (level $l=1): p_{i 1}=$ $0.3\left(1-p_{i 3}\right)$

Probability of minor disruption (level $l=2): p_{i 2}=$ $0.6\left(1-p_{i 3}\right)$ for all suppliers $i \in I$

Thus, $p_{i 0} \leq p_{i 1} \leq p_{i 2} \leq p_{i 3}$, which reflects a realworld relation among probabilities of disruption occurrence at different levels (e.g., [8]).

Regional disruption probabilities are $p^{1}=0.001$ and $p^{2}=$ 0.01 .

The probability of realizing of disruption scenario $s$ for suppliers in region $r=1,2$ is calculated as follows:

$$
\begin{aligned}
& P_{s}^{r} \\
& =\left\{\begin{array}{l}
\left(1-p^{r}\right)\left(\prod_{i \in I^{r}: \lambda_{i s}=0} 0.1\left(1-p_{i 3}\right)\right)\left(\prod_{i \in I^{r}: \lambda_{i s}=1} 0.3\left(1-p_{i 3}\right)\right)\left(\prod_{i \in I^{r}: \lambda_{i s}=2} 0.6\left(1-p_{i 3}\right)\right)\left(\prod_{i \in I^{r}: \lambda_{i s}=3} p_{i 3}\right), \quad \text { if } \sum_{i \in I^{r}} \lambda_{i s}>0, \\
p^{r}+\left(1-p^{r}\right) \prod_{i \in I^{r}} 0.1\left(1-p_{i 3}\right),
\end{array}\right.
\end{aligned}
$$

and the probability for disruption scenario $s \in S$ is given by $P_{s}=P_{s}^{1} P_{s}^{2}$.

Figure 2 presents basic characteristics of all suppliers: probability of complete shutdown, $p^{r}+\left(1-p^{r}\right) p_{i 0}, i \in I^{r}, r \in$ $R$, expected fulfillment rate, $\Gamma_{i}=\sum_{l=1,2,3}\left(1-p^{r}\right) p_{i l} \gamma_{i l}, i \in$ $I^{r}, r \in R$, and purchasing price, $o_{i}, i \in I$.

Table 2 presents a subset of nondominated solutions for the wait-and-see problem WCS, for a subset of trade-off parameter $\alpha \in\{0,0.1,0.2,0.3,0.4,0.5,0.6,0.7,0.8,0.9,1\}$. The results indicate that most of nondominated supply portfolios consist of the two suppliers only: the second most expensive and most reliable supplier $i=2$ from region $r=1$ and the cheapest and most unreliable supplier $i=3$ from region $r=2$. The most expensive and most reliable supplier $i=1$ in region $r=1$ and the second cheapest and most unreliable supplier $i=4$ in region $r=2$ are rarely selected. For $\alpha=1$ (minimization of cost), the cheapest supplier $i=3$ is selected only. For $\alpha=0$ (maximization of service level), the total demand for parts is allocated among the two most reliable and most expensive suppliers $i=1,2$. The above solution shows that the service-oriented supply portfolio ( $\alpha$ close to 0 ) is more diversified than the cost-oriented portfolio ( $\alpha$ close to 1 ). Table 2 also shows the associated expected fraction of fulfilled demand, $E_{3}=\sum_{s \in S} \sum_{j \in J} \sum_{t \in T} P_{s} b_{j} w_{j t}^{s} / B$, that is, demand fulfilled on time or delayed. The solution results demonstrate that a large expected service level is sometimes associated with a small expected fraction of fulfilled demand. Thus, the maximization of expected service level leads to rejection of customer orders that cannot be fulfilled by customer requested due dates.

Figure 3 shows the expected supply, production, and shipping schedules, respectively, for $\alpha=0$ (i.e., for the 

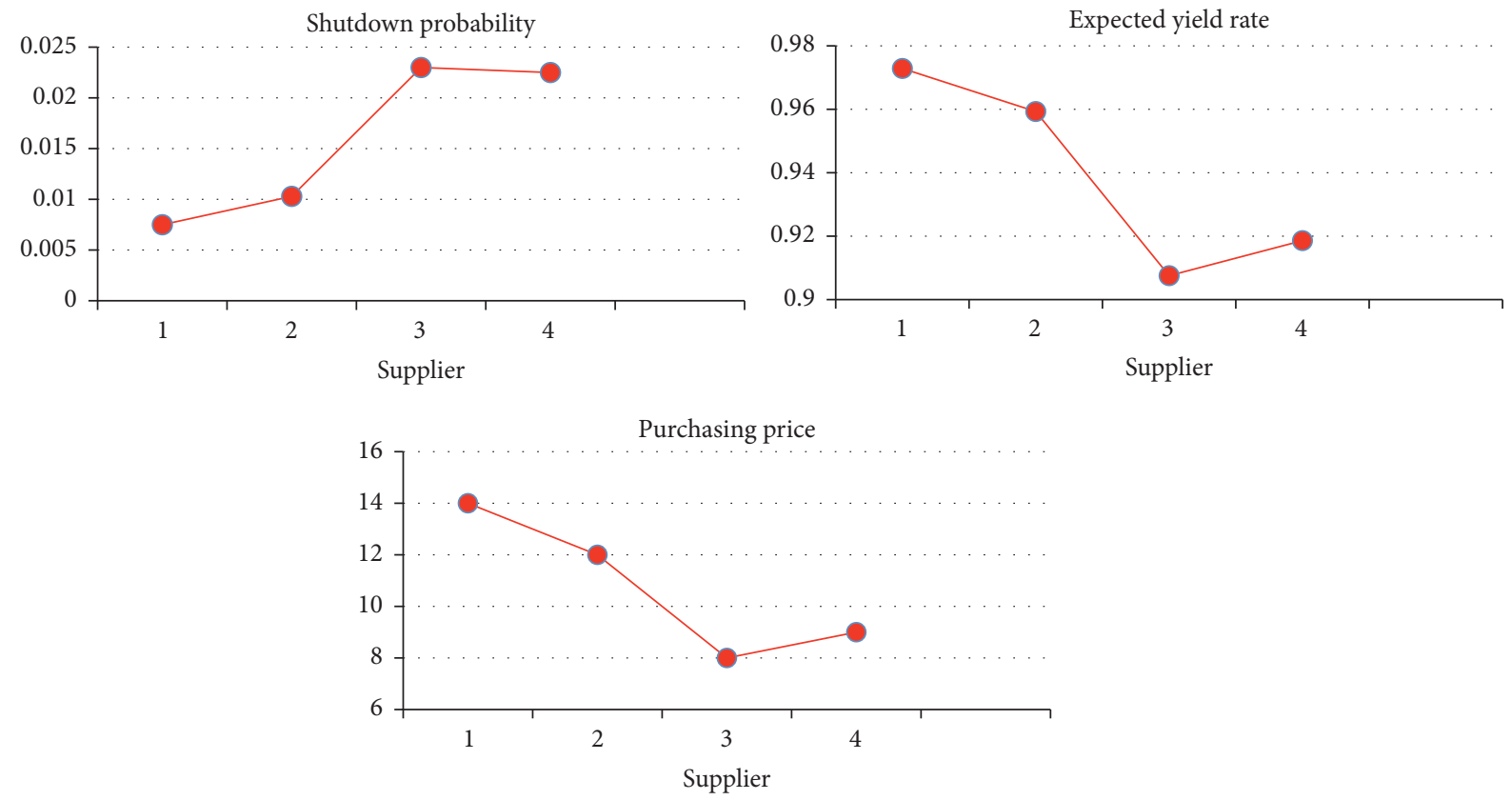

FIgURE 2: Suppliers.
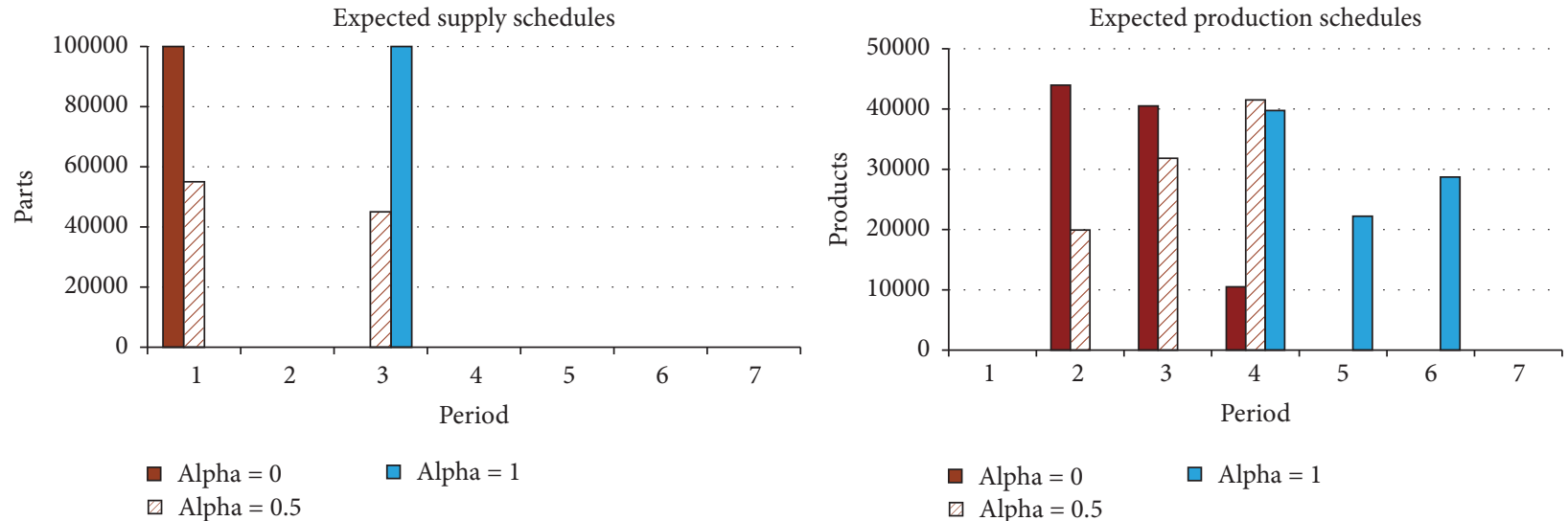

- Alpha $=0$

๑ Alpha $=0.5$

$$
\square \text { Alpha }=1
$$

๑ Alpha $=0.5$

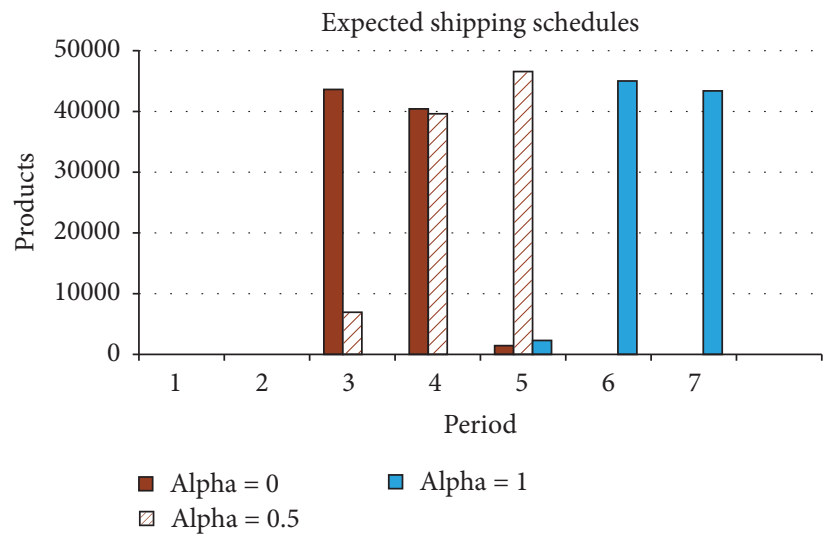

Figure 3: Expected schedules for model WCS. 
TABLE 1: Disruption scenarios.

\begin{tabular}{|c|c|c|c|c|}
\hline$s$ & $i=1$ & 2 & 3 & 4 \\
\hline 1 & 0 & 0 & 0 & 0 \\
\hline 2 & 0 & 0 & 0 & 1 \\
\hline 3 & 0 & 0 & 0 & 2 \\
\hline 4 & 0 & 0 & 0 & 3 \\
\hline 5 & 0 & 0 & 1 & 0 \\
\hline 6 & 0 & 0 & 1 & 1 \\
\hline 7 & 0 & 0 & 1 & 2 \\
\hline 8 & 0 & 0 & 1 & 3 \\
\hline 9 & 0 & 0 & 2 & 0 \\
\hline 10 & 0 & 0 & 2 & 1 \\
\hline 11 & 0 & 0 & 2 & 2 \\
\hline 12 & 0 & 0 & 2 & 3 \\
\hline 13 & 0 & 0 & 3 & 0 \\
\hline 14 & 0 & 0 & 3 & 1 \\
\hline 15 & 0 & 0 & 3 & 2 \\
\hline 16 & 0 & 0 & 3 & 3 \\
\hline 17 & 0 & 1 & 0 & 0 \\
\hline 18 & 0 & 1 & 0 & 1 \\
\hline 19 & 0 & 1 & 0 & 2 \\
\hline 20 & 0 & 1 & 0 & 3 \\
\hline 21 & 0 & 1 & 1 & 0 \\
\hline 22 & 0 & 1 & 1 & 1 \\
\hline 23 & 0 & 1 & 1 & 2 \\
\hline 24 & 0 & 1 & 1 & 3 \\
\hline 25 & 0 & 1 & 2 & 0 \\
\hline 26 & 0 & 1 & 2 & 1 \\
\hline 27 & 0 & 1 & 2 & 2 \\
\hline 28 & 0 & 1 & 2 & 3 \\
\hline 29 & 0 & 1 & 3 & 0 \\
\hline 30 & 0 & 1 & 3 & 1 \\
\hline 31 & 0 & 1 & 3 & 2 \\
\hline 32 & 0 & 1 & 3 & 3 \\
\hline 33 & 0 & 2 & 0 & 0 \\
\hline 34 & 0 & 2 & 0 & 1 \\
\hline 35 & 0 & 2 & 0 & 2 \\
\hline 36 & 0 & 2 & 0 & 3 \\
\hline 37 & 0 & 2 & 1 & 0 \\
\hline 38 & 0 & 2 & 1 & 1 \\
\hline 39 & 0 & 2 & 1 & 2 \\
\hline 40 & 0 & 2 & 1 & 3 \\
\hline 41 & 0 & 2 & 2 & 0 \\
\hline 42 & 0 & 2 & 2 & 1 \\
\hline 43 & 0 & 2 & 2 & 2 \\
\hline 44 & 0 & 2 & 2 & 3 \\
\hline 45 & 0 & 2 & 3 & 0 \\
\hline 46 & 0 & 2 & 3 & 1 \\
\hline 47 & 0 & 2 & 3 & 2 \\
\hline 48 & 0 & 2 & 3 & 3 \\
\hline 49 & 0 & 3 & 0 & 0 \\
\hline
\end{tabular}

TABLE 1: Continued.

\begin{tabular}{|c|c|c|c|c|}
\hline$s$ & $i=1$ & 2 & 3 & 4 \\
\hline 50 & 0 & 3 & 0 & 1 \\
\hline 51 & 0 & 3 & 0 & 2 \\
\hline 52 & 0 & 3 & 0 & 3 \\
\hline 53 & 0 & 3 & 1 & 0 \\
\hline 54 & 0 & 3 & 1 & 1 \\
\hline 55 & 0 & 3 & 1 & 2 \\
\hline 56 & 0 & 3 & 1 & 3 \\
\hline 57 & 0 & 3 & 2 & 0 \\
\hline 58 & 0 & 3 & 2 & 1 \\
\hline 59 & 0 & 3 & 2 & 2 \\
\hline 60 & 0 & 3 & 2 & 3 \\
\hline 61 & 0 & 3 & 3 & 0 \\
\hline 62 & 0 & 3 & 3 & 1 \\
\hline 63 & 0 & 3 & 3 & 2 \\
\hline 64 & 0 & 3 & 3 & 3 \\
\hline 65 & 1 & 0 & 0 & 0 \\
\hline 66 & 1 & 0 & 0 & 1 \\
\hline 67 & 1 & 0 & 0 & 2 \\
\hline 68 & 1 & 0 & 0 & 3 \\
\hline 69 & 1 & 0 & 1 & 0 \\
\hline 70 & 1 & 0 & 1 & 1 \\
\hline 71 & 1 & 0 & 1 & 2 \\
\hline 72 & 1 & 0 & 1 & 3 \\
\hline 73 & 1 & 0 & 2 & 0 \\
\hline 74 & 1 & 0 & 2 & 1 \\
\hline 75 & 1 & 0 & 2 & 2 \\
\hline 76 & 1 & 0 & 2 & 3 \\
\hline 77 & 1 & 0 & 3 & 0 \\
\hline 78 & 1 & 0 & 3 & 1 \\
\hline 79 & 1 & 0 & 3 & 2 \\
\hline 80 & 1 & 0 & 3 & 3 \\
\hline 81 & 1 & 1 & 0 & 0 \\
\hline 82 & 1 & 1 & 0 & 1 \\
\hline 83 & 1 & 1 & 0 & 2 \\
\hline 84 & 1 & 1 & 0 & 3 \\
\hline 85 & 1 & 1 & 1 & 0 \\
\hline 86 & 1 & 1 & 1 & 1 \\
\hline 87 & 1 & 1 & 1 & 2 \\
\hline 88 & 1 & 1 & 1 & 3 \\
\hline 89 & 1 & 1 & 2 & 0 \\
\hline 90 & 1 & 1 & 2 & 1 \\
\hline 91 & 1 & 1 & 2 & 2 \\
\hline 92 & 1 & 1 & 2 & 3 \\
\hline 93 & 1 & 1 & 3 & 0 \\
\hline 94 & 1 & 1 & 3 & 1 \\
\hline 95 & 1 & 1 & 3 & 2 \\
\hline 96 & 1 & 1 & 3 & 3 \\
\hline 97 & 1 & 2 & 0 & 0 \\
\hline 98 & 1 & 2 & 0 & 1 \\
\hline
\end{tabular}


TABle 1: Continued.

\begin{tabular}{|c|c|c|c|c|}
\hline$s$ & $i=1$ & 2 & 3 & 4 \\
\hline 99 & 1 & 2 & 0 & 2 \\
\hline 100 & 1 & 2 & 0 & 3 \\
\hline 101 & 1 & 2 & 1 & 0 \\
\hline 102 & 1 & 2 & 1 & 1 \\
\hline 103 & 1 & 2 & 1 & 2 \\
\hline 104 & 1 & 2 & 1 & 3 \\
\hline 105 & 1 & 2 & 2 & 0 \\
\hline 106 & 1 & 2 & 2 & 1 \\
\hline 107 & 1 & 2 & 2 & 2 \\
\hline 108 & 1 & 2 & 2 & 3 \\
\hline 109 & 1 & 2 & 3 & 0 \\
\hline 110 & 1 & 2 & 3 & 1 \\
\hline 111 & 1 & 2 & 3 & 2 \\
\hline 112 & 1 & 2 & 3 & 3 \\
\hline 113 & 1 & 3 & 0 & 0 \\
\hline 114 & 1 & 3 & 0 & 1 \\
\hline 115 & 1 & 3 & 0 & 2 \\
\hline 116 & 1 & 3 & 0 & 3 \\
\hline 117 & 1 & 3 & 1 & 0 \\
\hline 118 & 1 & 3 & 1 & 1 \\
\hline 119 & 1 & 3 & 1 & 2 \\
\hline 120 & 1 & 3 & 1 & 3 \\
\hline 121 & 1 & 3 & 2 & 0 \\
\hline 122 & 1 & 3 & 2 & 1 \\
\hline 123 & 1 & 3 & 2 & 2 \\
\hline 124 & 1 & 3 & 2 & 3 \\
\hline 125 & 1 & 3 & 3 & 0 \\
\hline 126 & 1 & 3 & 3 & 1 \\
\hline 127 & 1 & 3 & 3 & 2 \\
\hline 128 & 1 & 3 & 3 & 3 \\
\hline 129 & 2 & 0 & 0 & 0 \\
\hline 130 & 2 & 0 & 0 & 1 \\
\hline 131 & 2 & 0 & 0 & 2 \\
\hline 132 & 2 & 0 & 0 & 3 \\
\hline 133 & 2 & 0 & 1 & 0 \\
\hline 134 & 2 & 0 & 1 & 1 \\
\hline 135 & 2 & 0 & 1 & 2 \\
\hline 136 & 2 & 0 & 1 & 3 \\
\hline 137 & 2 & 0 & 2 & 0 \\
\hline 138 & 2 & 0 & 2 & 1 \\
\hline 139 & 2 & 0 & 2 & 2 \\
\hline 140 & 2 & 0 & 2 & 3 \\
\hline 141 & 2 & 0 & 3 & 0 \\
\hline 142 & 2 & 0 & 3 & 1 \\
\hline 143 & 2 & 0 & 3 & 2 \\
\hline 144 & 2 & 0 & 3 & 3 \\
\hline 145 & 2 & 1 & 0 & 0 \\
\hline 146 & 2 & 1 & 0 & 1 \\
\hline 147 & 2 & 1 & 0 & 2 \\
\hline 148 & 2 & 1 & 0 & 3 \\
\hline 149 & 2 & 1 & 1 & 0 \\
\hline 150 & 2 & 1 & 1 & 1 \\
\hline
\end{tabular}

TABLE 1: Continued.

\begin{tabular}{|c|c|c|c|c|}
\hline$s$ & $i=1$ & 2 & 3 & 4 \\
\hline 151 & 2 & 1 & 1 & 2 \\
\hline 152 & 2 & 1 & 1 & 3 \\
\hline 153 & 2 & 1 & 2 & 0 \\
\hline 154 & 2 & 1 & 2 & 1 \\
\hline 155 & 2 & 1 & 2 & 2 \\
\hline 156 & 2 & 1 & 2 & 3 \\
\hline 157 & 2 & 1 & 3 & 0 \\
\hline 158 & 2 & 1 & 3 & 1 \\
\hline 159 & 2 & 1 & 3 & 2 \\
\hline 160 & 2 & 1 & 3 & 3 \\
\hline 161 & 2 & 2 & 0 & 0 \\
\hline 162 & 2 & 2 & 0 & 1 \\
\hline 163 & 2 & 2 & 0 & 2 \\
\hline 164 & 2 & 2 & 0 & 3 \\
\hline 165 & 2 & 2 & 1 & 0 \\
\hline 166 & 2 & 2 & 1 & 1 \\
\hline 167 & 2 & 2 & 1 & 2 \\
\hline 168 & 2 & 2 & 1 & 3 \\
\hline 169 & 2 & 2 & 2 & 0 \\
\hline 170 & 2 & 2 & 2 & 1 \\
\hline 171 & 2 & 2 & 2 & 2 \\
\hline 172 & 2 & 2 & 2 & 3 \\
\hline 173 & 2 & 2 & 3 & 0 \\
\hline 174 & 2 & 2 & 3 & 1 \\
\hline 175 & 2 & 2 & 3 & 2 \\
\hline 176 & 2 & 2 & 3 & 3 \\
\hline 177 & 2 & 3 & 0 & 0 \\
\hline 178 & 2 & 3 & 0 & 1 \\
\hline 179 & 2 & 3 & 0 & 2 \\
\hline 180 & 2 & 3 & 0 & 3 \\
\hline 181 & 2 & 3 & 1 & 0 \\
\hline 182 & 2 & 3 & 1 & 1 \\
\hline 183 & 2 & 3 & 1 & 2 \\
\hline 184 & 2 & 3 & 1 & 3 \\
\hline 185 & 2 & 3 & 2 & 0 \\
\hline 186 & 2 & 3 & 2 & 1 \\
\hline 187 & 2 & 3 & 2 & 2 \\
\hline 188 & 2 & 3 & 2 & 3 \\
\hline 189 & 2 & 3 & 3 & 0 \\
\hline 190 & 2 & 3 & 3 & 1 \\
\hline 191 & 2 & 3 & 3 & 2 \\
\hline 192 & 2 & 3 & 3 & 3 \\
\hline 193 & 3 & 0 & 0 & 0 \\
\hline 194 & 3 & 0 & 0 & 1 \\
\hline 195 & 3 & 0 & 0 & 2 \\
\hline 196 & 3 & 0 & 0 & 3 \\
\hline 197 & 3 & 0 & 1 & 0 \\
\hline 198 & 3 & 0 & 1 & 1 \\
\hline 199 & 3 & 0 & 1 & 2 \\
\hline 200 & 3 & 0 & 1 & 3 \\
\hline 201 & 3 & 0 & 2 & 0 \\
\hline 202 & 3 & 0 & 2 & 1 \\
\hline
\end{tabular}


TABle 1: Continued.

\begin{tabular}{|c|c|c|c|c|}
\hline$s$ & $i=1$ & 2 & 3 & 4 \\
\hline 203 & 3 & 0 & 2 & 2 \\
\hline 204 & 3 & 0 & 2 & 3 \\
\hline 205 & 3 & 0 & 3 & 0 \\
\hline 206 & 3 & 0 & 3 & 1 \\
\hline 207 & 3 & 0 & 3 & 2 \\
\hline 208 & 3 & 0 & 3 & 3 \\
\hline 209 & 3 & 1 & 0 & 0 \\
\hline 210 & 3 & 1 & 0 & 1 \\
\hline 211 & 3 & 1 & 0 & 2 \\
\hline 212 & 3 & 1 & 0 & 3 \\
\hline 213 & 3 & 1 & 1 & 0 \\
\hline 214 & 3 & 1 & 1 & 1 \\
\hline 215 & 3 & 1 & 1 & 2 \\
\hline 216 & 3 & 1 & 1 & 3 \\
\hline 217 & 3 & 1 & 2 & 0 \\
\hline 218 & 3 & 1 & 2 & 1 \\
\hline 219 & 3 & 1 & 2 & 2 \\
\hline 220 & 3 & 1 & 2 & 3 \\
\hline 221 & 3 & 1 & 3 & 0 \\
\hline 222 & 3 & 1 & 3 & 1 \\
\hline 223 & 3 & 1 & 3 & 2 \\
\hline 224 & 3 & 1 & 3 & 3 \\
\hline 225 & 3 & 2 & 0 & 0 \\
\hline 226 & 3 & 2 & 0 & 1 \\
\hline 227 & 3 & 2 & 0 & 2 \\
\hline 228 & 3 & 2 & 0 & 3 \\
\hline 229 & 3 & 2 & 1 & 0 \\
\hline 230 & 3 & 2 & 1 & 1 \\
\hline 231 & 3 & 2 & 1 & 2 \\
\hline 232 & 3 & 2 & 1 & 3 \\
\hline 233 & 3 & 2 & 2 & 0 \\
\hline 234 & 3 & 2 & 2 & 1 \\
\hline 235 & 3 & 2 & 2 & 2 \\
\hline 236 & 3 & 2 & 2 & 3 \\
\hline 237 & 3 & 2 & 3 & 0 \\
\hline 238 & 3 & 2 & 3 & 1 \\
\hline 239 & 3 & 2 & 3 & 2 \\
\hline 240 & 3 & 2 & 3 & 3 \\
\hline 241 & 3 & 3 & 0 & 0 \\
\hline 242 & 3 & 3 & 0 & 1 \\
\hline 243 & 3 & 3 & 0 & 2 \\
\hline 244 & 3 & 3 & 0 & 3 \\
\hline 245 & 3 & 3 & 1 & 0 \\
\hline 246 & 3 & 3 & 1 & 1 \\
\hline 247 & 3 & 3 & 1 & 2 \\
\hline 248 & 3 & 3 & 1 & 3 \\
\hline 249 & 3 & 3 & 2 & 0 \\
\hline 250 & 3 & 3 & 2 & 1 \\
\hline 251 & 3 & 3 & 2 & 2 \\
\hline 252 & 3 & 3 & 2 & 3 \\
\hline
\end{tabular}

TABLE 1: Continued.

\begin{tabular}{lclll}
\hline$s$ & $i=1$ & 2 & 3 & 4 \\
\hline 253 & 3 & 3 & 3 & 0 \\
254 & 3 & 3 & 3 & 1 \\
255 & 3 & 3 & 3 & 2 \\
256 & 3 & 3 & 3 & 3 \\
\hline
\end{tabular}

maximum expected service level), $\alpha=0.5$, and $\alpha=1$ (i.e., for the minimum expected cost). The expected schedules were computed using the formulae presented below:

(i) Expected schedule of supplies of parts to the producer:

$$
\sum_{s \in S} \sum_{i \in I_{s}: \sigma_{i}=t} P_{s} B \gamma_{i, \lambda_{i s}} v_{i} ; \quad t \in T
$$

(ii) Expected production schedule:

$$
\sum_{s \in S} \sum_{j \in J} P_{s} b_{j} w_{j t}^{s} ; \quad t \in T
$$

(iii) Expected schedule of shipping of products from the producer to the distribution centers:

$$
\sum_{s \in S} \sum_{k \in K} \sum_{j \in J_{k}} P_{s} b_{j}\left(\sum_{t^{\prime} \in T: t^{\prime}<t} w_{j t^{\prime}}^{s}\right) x_{k t}^{s} ; \quad t \in T
$$

As $\alpha$ increases, that is, the decision-maker's priority shifts from the maximum service level to minimum cost and more parts are ordered from less reliable and lower cost suppliers, the expected supply schedules and the corresponding production schedules are more delayed as well as the delivery of products to the customers. Note that, despite constraint (13) that ensures a feasible schedule with at most one shipment of products to each distribution center for each disruption scenario, the expected shipping schedule (29) may be split into more smaller size shipments (cf. Figure 3, where two major and one small-size shipments are indicated for each confidence level).

For comparison, Table 3 presents a subset of nondominated solutions obtained for the expected value problem EWCS, and Figure 4 shows supply, production, and shipping schedules. Unlike the stochastic programming approach which accounts for all potential disruption scenarios to optimize an expected performance of the supply chain, the solution obtained using the deterministic approach is based on aggregate information on suppliers expected fulfillment. In general, the results are similar for both models and the corresponding optimal solution values are close to each other, which indicates that the expected value problem can be used in practice, when stochastic mixed integer programs are hard to solve. The optimal solution values for the expected value problem frequently outperform the corresponding solution values of the wait-and-see problem, which is in line with the proposition EV $\leq \mathrm{WS}$, in [27]; compare Section 5, for example, the minimum cost $\bar{E}_{1}$ for EWCS (see Table 3), 
TABLE 2: Nondominated solutions for model WCS.

\begin{tabular}{|c|c|c|c|c|c|c|c|c|c|}
\hline$\alpha$ & 0 & 0.1 & 0.2 & 0.3 & 0.4 & 0.5 and 0.6 & 0.7 & 0.8 and 0.9 & 1 \\
\hline \multicolumn{10}{|c|}{ Var. $=38077 ;$ Bin. $=33076 ;$ Cons. $=28769 ;$ Nonz. $=348325^{(\mathrm{a})}$} \\
\hline Exp. cost & $17.31\left(\bar{E}_{1}\right)$ & 16.20 & 15.47 & 12.06 & 12.00 & 11.76 & 10.70 & 10.65 & $10.51\left(\underline{E}_{1}\right)$ \\
\hline Exp. service level ${ }^{(b)}$ & $85.52\left(\bar{E}_{2}\right)$ & 84.87 & 84.52 & 77.19 & 76.76 & 75.95 & 58.63 & 58.71 & $38.22\left(\underline{E}_{2}\right)$ \\
\hline Exp. fulfilled demand ${ }^{(c)}$ & 85.52 & 85.30 & 84.52 & 93.70 & 93.61 & 93.53 & 91.34 & 91.20 & 90.68 \\
\hline \multirow{4}{*}{$\begin{array}{l}\text { Suppliers selected } \\
\text { (\% of total demand) }\end{array}$} & $1(76)$ & $1(35)$ & & & & & & & \\
\hline & $2(24)$ & $2(55)$ & $2(87)$ & $2(55)$ & $2(55)$ & $2(55)$ & $2(12)$ & $2(10)$ & \\
\hline & & $3(10)$ & $3(13)$ & $3(22)$ & $3(32)$ & $3(45)$ & $3(88)$ & $3(90)$ & $3(100)$ \\
\hline & & & & $4(23)$ & $4(13)$ & & & & \\
\hline
\end{tabular}

$\overline{{ }^{(a)} \text { Var.: number of variables; Bin.: number of binary variables; Cons.: number of constraints; Nonz.: number of nonzero coefficients. }{ }^{(\mathrm{b})} \sum_{s \in S} \sum_{j \in J} P_{s} b_{j} y_{j}^{s} / B \times}$ $100 \%{ }^{(\mathrm{c})} \sum_{s \in S} \sum_{j \in J} \sum_{t \in T} P_{s} b_{j} w_{j t}^{s} / B \times 100 \%$.

TABLE 3: Nondominated solutions for model EWCS.

\begin{tabular}{lccccc}
\hline$\alpha$ & 0 & $0.1,0.2$ and 0.3 & 0.4 and 0.5 & 0.6 and 0.7 & $0.8,0.9$ and 1 \\
& \multicolumn{2}{c}{ Var. = 162; Bin. $=136$; Cons. $=125$; Nonz. $=1430^{(\mathrm{a})}$} & 12.14 & 11.67 & $10.45\left(\underline{E}_{1}\right)$ \\
\hline Exp. cost & $16.91\left(\bar{E}_{1}\right)$ & 14.27 & 81 & 79 & $62\left(\underline{E}_{2}\right)$ \\
Exp. service level $^{(\mathrm{b})}$ & $87\left(\bar{E}_{2}\right)$ & 86 & 91 & 93 \\
Exp. fulfilled demand $^{(\mathrm{c})}$ & 87 & 90 & $2(49)$ & $2(50)$ & $3(50)$ \\
\hline & $1(89)$ & $2(94)$ & $3(51)$ & $3(95)$ \\
Suppliers selected & & $3(6)$ & & & $3(50)$ \\
\hline
\end{tabular}

${ }^{(a)}$ Var.: number of variables; Bin.: number of binary variables; Cons.: number of constraints; Nonz.: number of nonzero coefficients. ${ }^{\text {(b) }} \sum_{j \in J} b_{j} Y_{j} / B \times 100 \%$. ${ }^{(\mathrm{c})} \sum_{j \in J} \sum_{t \in T} b_{j} W_{j t} / B \times 100 \%$.
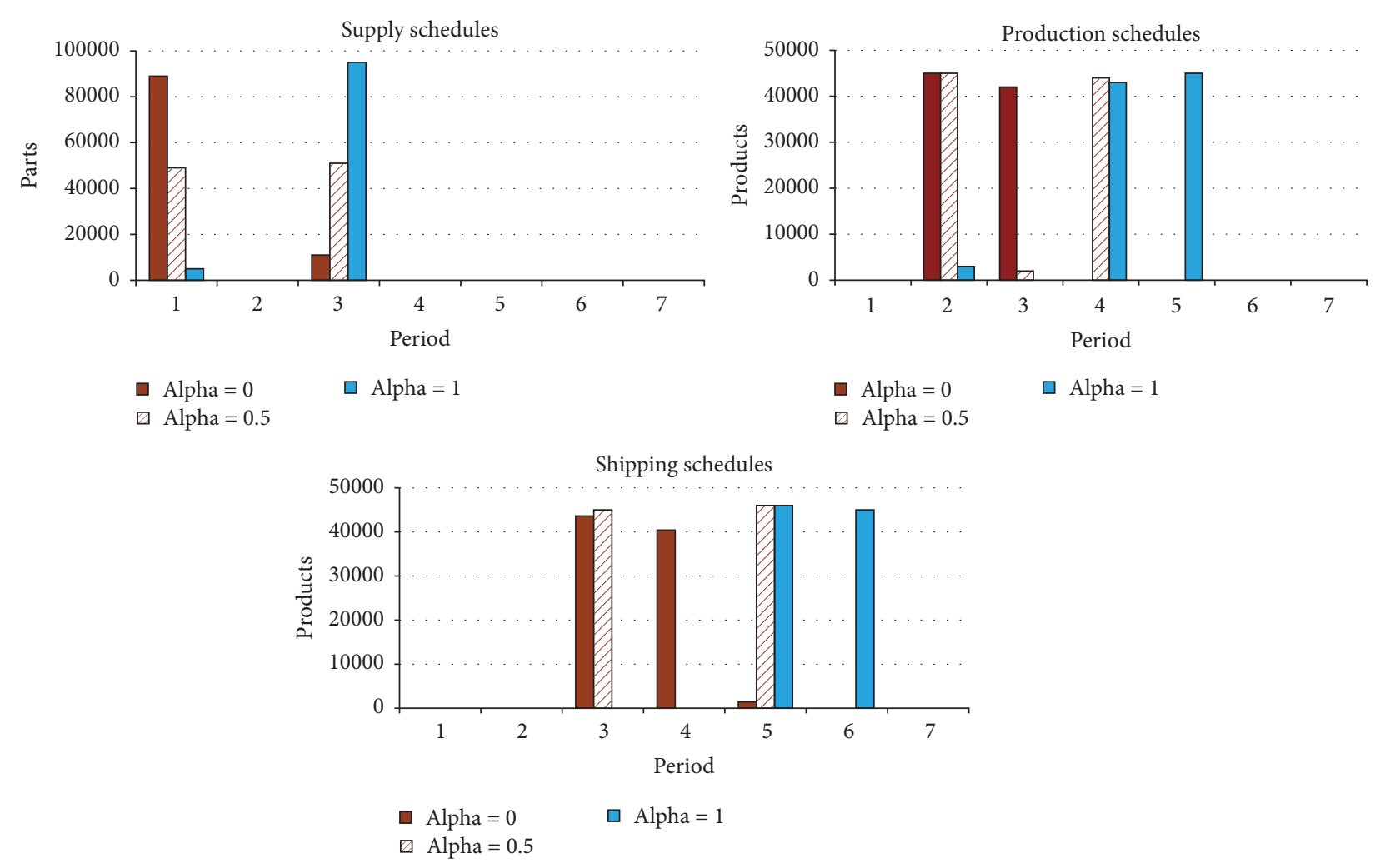

FIGURE 4: Schedules for model EWCS. 
$10.45 \leq 10.51$ for $W C S$ (see Table 2), or the maximum service level $\bar{E}_{2} \%$ for $E W C S$ (see Table 3), $87.00 \geq 85.52$ for $W C S$ (see Table 2).

The stochastic wait-and-see approach, however, leads to a more diversified supply portfolio. For the deterministic approach, most of nondominated supply portfolios consist of two suppliers only: $i=2,3$, while suppliers $i=1,4$ are selected only for the maximum service level objective (that is, for $\alpha=0$ ). Comparison of Figures 3 and 4 indicates that the expected schedules for model WCS, computed as expectations over all schedules for all potential disruption scenarios, (27)-(29), are similar to the corresponding single schedules determined by model EWCS. The main differences observed are more delayed expected production and shipping schedules for model WCS when minimization of cost is considered (that is, for $\alpha=1$ ). Finally, it is interesting to note that, in the multiple sourcing environment considered, both the wait-and-see approach and, in particular, the expected value approach frequently select a dual sourcing supply portfolio, with one main supplier and one supporting supplier.

The computational experiments were performed using the AMPL programming language and the CPLEX 12.6.2 solver on a MacBook Pro laptop with Intel Core i7 processor running at $2.8 \mathrm{GHz}$ and with $16 \mathrm{~GB}$ RAM. The solver was capable of finding proven optimal solution for all examples with CPU time ranging from several minutes to several hours for the stochastic model WCS and fraction of a second for the deterministic model EWCS. Since the stochastic programming model WCS needs to determine nondominated schedules for all potential disruption scenarios, while model EWCS deals with a single scenario only, the difference in the computational effort required is obvious.

\section{Conclusions}

In this paper, two biobjective MIP formulations, stochastic and deterministic, have been proposed and compared for the coordinated decision-making in supply chains under partial local disruptions and all-or-nothing regional disruptions. The problem objective has been to jointly schedule supplies, production, and distribution to optimize the trade-off between expected cost and expected service level. While the stochastic programming approach aims at optimizing the expected performance of a supply chain over all possible disruption scenarios, the deterministic approach accounts only for a single scenario representing average disruption conditions. As a result, the stochastic programming approach determines nondominated solutions for all disruption scenarios, whereas the deterministic approach produces a single solution only. In particular, in the stochastic programming approach, the selection of supply portfolio is combined with supply chain scheduling for all disruption scenarios considered. In contrast, the deterministic expected value approach provides the portfolio along with a single expected schedule of production and distribution.

The expected schedules obtained for the stochastic programming model WCS, as expectations over all schedules for all disruption scenarios, have been compared with the corresponding schedules determined by the deterministic model EWCS, based on an expected disruption scenario. The comparison has demonstrated that the two approaches lead to similar expected solutions.

The main findings are in line with other research and are listed as follows:

(i) The two decision-making approaches, stochastic and deterministic, lead to similar expected performance of a supply chain under multilevel disruptions.

(ii) The optimal solution values for the expected value problem frequently outperform the corresponding solution values of the wait-and-see problem.

(iii) Despite the multiple sourcing environment considered, both the wait-and-see approach and, in particular, the expected value approach frequently select a dual sourcing supply portfolio, with one main supplier and one supporting supplier.

(iv) The stochastic approach, which accounts for all potential disruption scenarios, may lead to a more diversified supply portfolio that will hedge against a variety of scenarios.

(v) The expected schedules are more delayed for the stochastic approach.

(vi) The service-oriented supply portfolio is more diversified and may combine both high-cost, reliable suppliers and low-cost unreliable suppliers, while the costoriented portfolio depends mainly on low-cost and less reliable suppliers.

Overall, the results of computational experiments indicate that the proposed approach and developed MIP models are flexible and efficient tools for coordinated supply chain scheduling. The portfolio approach leads to MIP formulations with strong LP relaxations and has been proven to be computationally very efficient. CPU time required to find proven optimal solutions for realistic size examples, using commercially available software for MIP, is acceptable for a real-world supply chain disruption management (see Sawik [33]).

Since the probability distribution of supply disruptions from each supplier is usually unknown (e.g., [8]), local multilevel disruptions in the stochastic programming model WCS have a multinomial discrete distribution, while the two-level regional disruptions have a binomial discrete distribution, and all disruption events are independent. As part of future research, we propose to enhance the stochastic programming model for more general scenarios with finitely many elements singled out and all the probability concentrated in them. For example, rather than complete shutdown, suppliers within a region may have correlated disruptions. Another important stream of future research is the study of robustness and sensitivity in relation to input data changes in supply chain scheduling under disruption risks (e.g., [33]). Future research can also focus on the following improvement of the proposed models: to consider the minimization of time and cost of recovery (e.g., Whitney et al. [34]). 


\section{Notations}

Indices

$i$ : Supplier, $i \in I$

$j$ : Customer, $j \in J$

$k$ : Distribution center, $k \in K$

$l$ : Disruption level, $l \in L$

$r$ : Region, $r \in R$

$s:$ Disruption scenario, $s \in S$

$t$ : Planning period, $t \in T$

\section{Input Parameters}

$b_{j}$ : Size (number of products) of customer order $j$

$B$ : Total demand for parts/products, $B=\sum_{j \in J} b_{j}$

$C$ : Capacity of producer

$d_{j}$ : Due date for customer order $j$

$e_{i}$ : Fixed cost of ordering parts from supplier $i$

$g_{j}$ : Per unit penalty cost of delayed customer order $j$

$h_{j}$ : Per unit penalty cost of unfulfilled customer order $j$

$I^{r}$ : Subset of suppliers in region $r$

$J_{k}$ : Subset of customers serviced by distribution center $k$

$o_{i}$ : Per unit price of parts purchased from supplier $i$

$p_{i l}$ : Probability of disruption level $l$ for supplier $i$

$p^{r}$ : Regional disruption probability for region $r$

$\gamma_{i l}$ : Fraction of an order delivered by supplier $i$ under disruption level $l$ (fulfillment rate)

$\Gamma_{i}$ : Expected fraction of an order delivered by supplier $i$ (expected fulfillment rate)

$\sigma_{i}$ : Delivery lead time from supplier $i$

$\tau_{k}$ : Transportation time to distribution center $k$.

\section{Conflicts of Interest}

The author declares that there are no conflicts of interest.

\section{Acknowledgments}

This work has been supported by NCN research grant (no. DEC-2013/11/B/ST8/04458) and by AGH (no. 11.11.200.324).

\section{References}

[1] J. V. Blackhurst, K. P. Scheibe, and D. J. Johnson, "Supplier risk assessment and monitoring for the automotive industry," International Journal of Physical Distribution \& Logistics Management, vol. 38, no. 2, pp. 143-165, 2008.

[2] P. Hoffmann, H. Schiele, and K. Krabbendam, "Uncertainty, supply risk management and their impact on performance," Journal of Purchasing \& Supply Management, vol. 19, no. 3, pp. 199-211, 2013.

[3] Y. Park, P. Hong, and J. J. Roh, "Supply chain lessons from the catastrophic natural disaster in Japan," Business Horizons, vol. 56, no. 1, pp. 75-85, 2013.

[4] M. Haraguchi and U. Lall, "Flood risks and impacts: A case study of Thailand's floods in 2011 and research questions for supply chain decision making," International Journal of Disaster Risk Reduction, vol. 14, pp. 256-272, 2015.
[5] T. Fujimoto and Y. W. Park, "Balancing supply chain competitiveness and robustness through "virtual dual sourcing": Lessons from the Great East Japan Earthquake," International Journal of Production Economics, vol. 147, pp. 429-436, 2014.

[6] H. Matsuo, "Implications of the Tohoku earthquake for Toyota's coordination mechanism: supply chain disruption of automotive semiconductors," International Journal of Production Economics, vol. 161, pp. 217-227, 2015.

[7] J. R. Marszewska, "Implications of seismic hazard in Japan on toyota supply chain disruption risks," in Proceedings of the 13th International Conference on Industrial Logistics, (ICIL '16), pp. 178-185, Zakopane, Poland, October 2016.

[8] A. Z. Zeng and Y. Xia, "Building a mutually beneficial partnership to ensure backup supply," Omega, vol. 52, pp. 77-91, 2015.

[9] Ş. S. Erengüç, N. C. Simpson, and A. J. Vakharia, "Integrated production/distribution planning in supply chains: an invited review," European Journal of Operational Research, vol. 115, no. 2, pp. 219-236, 1999.

[10] L. Lei, S. Liu, A. Ruszczynski, and S. Park, "On the integrated production, inventory, and distribution routing problem," IIE Transactions, vol. 38, no. 11, pp. 955-970, 2006.

[11] A. Kaur, A. Kanda, and S. G. Deshmukh, "A graph theoretic approach for supply chain coordination," International Journal of Logistics Systems and Management, vol. 2, no. 4, pp. 321-341, 2006.

[12] T. Choi, W. Yeung, and T. C. E. Cheng, "Scheduling and co-ordination of multi-suppliers single-warehouse-operator singlemanufacturer supply chains with variable production rates and storage costs," International Journal of Production Research, vol. 51, no. 9, pp. 2593-2601, 2013.

[13] S. Liu and L. G. Papageorgiou, "Multiobjective optimisation of production, distribution and capacity planning of global supply chains in the process industry," Omega, vol. 41, no. 2, pp. 369382, 2013.

[14] Z. L. Chen, "Integrated production and outbound distribution scheduling: review and extensions," Operations Research, vol. 58, no. 1, pp. 130-148, 2010.

[15] T. Sawik, "Coordinated supply chain scheduling," International Journal of Production Economics, vol. 120, no. 2, pp. 437-451, 2009.

[16] B. Tomlin, "On the value of mitigation and contingency strategies for managing supply chain disruption risks," Management Science, vol. 52, no. 5, pp. 639-657, 2006.

[17] D. Ivanov, B. Sokolov, and A. Dolgui, "The Ripple effect in supply chains: Trade-off 'efficiency-flexibility- resilience' in disruption management," International Journal of Production Research, vol. 52, no. 7, pp. 2154-2172, 2014.

[18] D. Ivanov, A. Pavlov, and B. Sokolov, "Optimal distribution (re)planning in a centralized multi-stage supply network under conditions of the ripple effect and structure dynamics," European Journal of Operational Research, vol. 237, no. 2, pp. 758770, 2014.

[19] D. Ivanov, A. Pavlov, A. Dolgui, D. Pavlov, and B. Sokolov, "Disruption-driven supply chain (re)-planning and performance impact assessment with consideration of pro-active and recovery policies," Transportation Research Part E: Logistics and Transportation Review, vol. 90, pp. 7-24, 2016.

[20] A. J. Schmitt and L. V. Snyder, "Infinite-horizon models for inventory control under yield uncertainty and disruptions," Computers \& Operations Research, vol. 39, no. 4, pp. 850-862, 2012. 
[21] A. J. Schmitt, S. A. Sun, L. V. Snyder, and Z.-J. M. Shen, “Centralization versus decentralization: Risk pooling, risk diversification, and supply chain disruptions," Omega, vol. 52, pp. 201212, 2015.

[22] L. V. Snyder, Z. Atan, P. Peng, Y. Rong, A. J. Schmitt, and B. Sinsoysal, "OR/MS models for supply chain disruptions: a review," IIE Transactions, vol. 48, no. 2, pp. 89-109, 2016.

[23] T. Sawik, "Integrated selection of suppliers and scheduling of customer orders in the presence of supply chain disruption risks," International Journal of Production Research, vol. 51, no. 23-24, pp. 7006-7022, 2013.

[24] T. Sawik, "Optimization of cost and service level in the presence of supply chain disruption risks: Single vs. multiple sourcing," Computers \& Operations Research, vol. 51, pp. 11-20, 2014.

[25] T. Sawik, "Integrated supply, production and distribution scheduling under disruption risks," Omega, vol. 62, pp. 131-144, 2016.

[26] T. Sawik, "Integrated supply chain scheduling under multi-level disruptions," IFAC-PapersOnLine, vol. 28, no. 3, pp. 1515-1520, 2015.

[27] P. Kall and J. Mayer, Stochastic Linear Programming: Models, Theory, and Computation, vol. 156 of International Series in Operations Research \& Management Science, Springer, New York, 2011.

[28] I. N. Durbach and T. J. Stewart, "Using expected values to simplify decision making under uncertainty," Omega, vol. 37, no. 2, pp. 312-330, 2009.

[29] F. Maggioni and S. W. Wallace, "Analyzing the quality of the expected value solution in stochastic programming," Annals of Operations Research, vol. 200, pp. 37-54, 2012.

[30] T. Sawik, "Selection of supply portfolio under disruption risks," Omega, vol. 39, no. 2, pp. 194-208, 2011.

[31] E. Delage, S. Arroyo, and Y. Ye, "The value of stochastic modeling in two-stage stochastic programs with cost uncertainty," Operations Research, vol. 62, no. 6, pp. 1377-1393, 2014.

[32] T. Sawik, Scheduling in Supply Chains Using Mixed Integer Programming, John Wiley \& Sons, Inc., Hoboken, NJ, 2011.

[33] T. Sawik, Supply Chain Disruption Management Using Stochastic Mixed Integer Programming, vol. 256 of International Series in Operations Research \& Management Science, Springer, New York, 2017, http://www.springer.com/gp/book/9783319588223.

[34] D. E. Whitney, J. Luo, and D. A. Heller, "The benefits and constraints of temporary sourcing diversification in supply chain disruption and recovery," Journal of Purchasing and Supply Management, vol. 20, no. 4, pp. 238-250, 2014. 


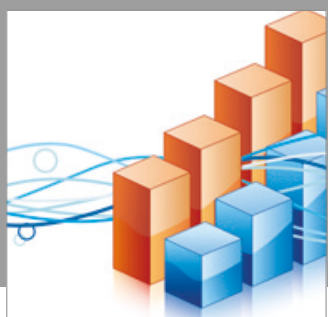

Advances in

Operations Research

vatersals

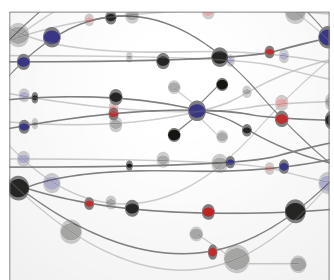

\section{The Scientific} World Journal
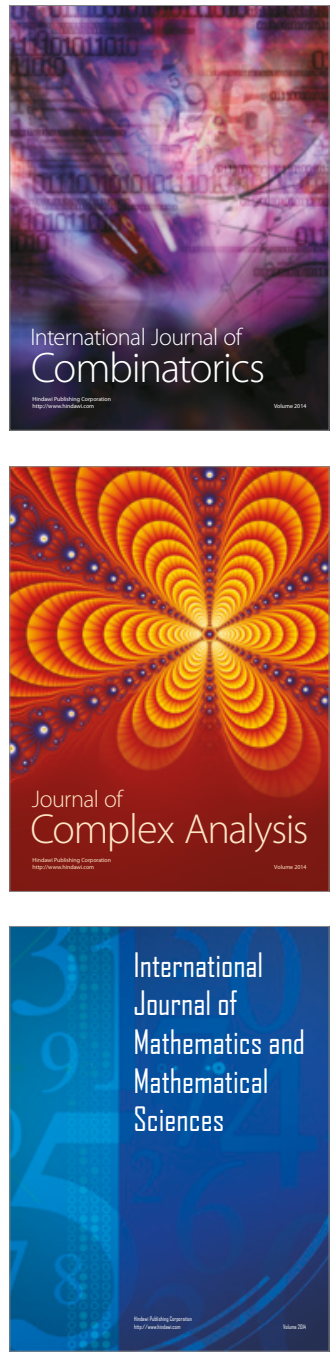
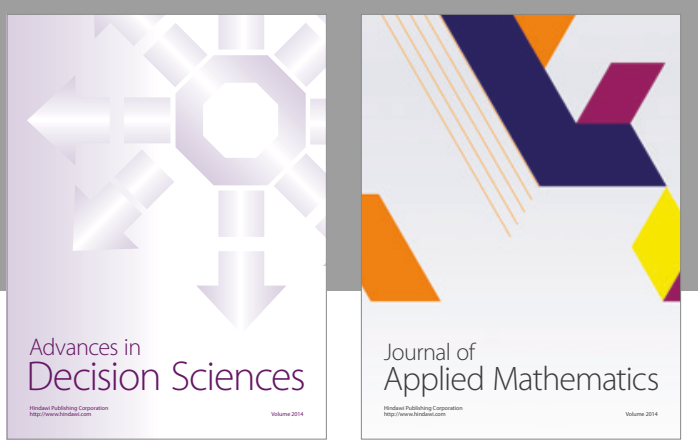

Algebra

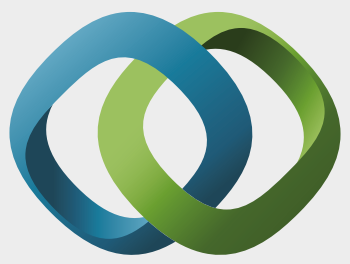

\section{Hindawi}

Submit your manuscripts at

https://www.hindawi.com
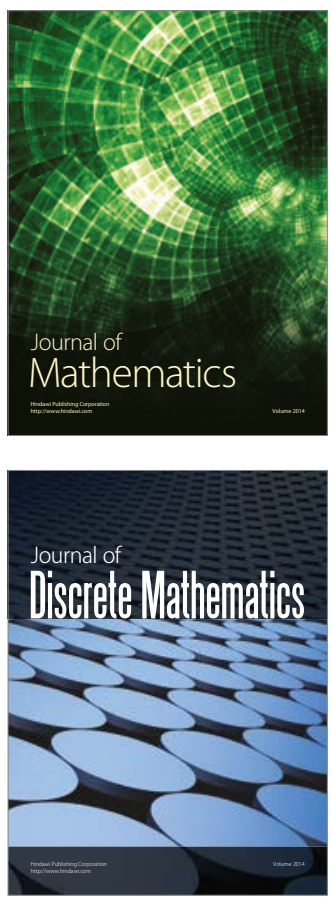

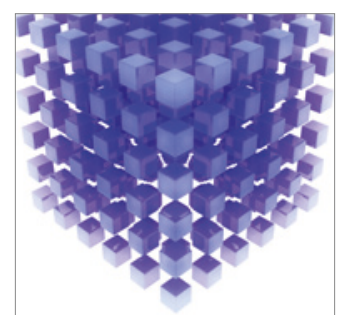

Mathematical Problems in Engineering
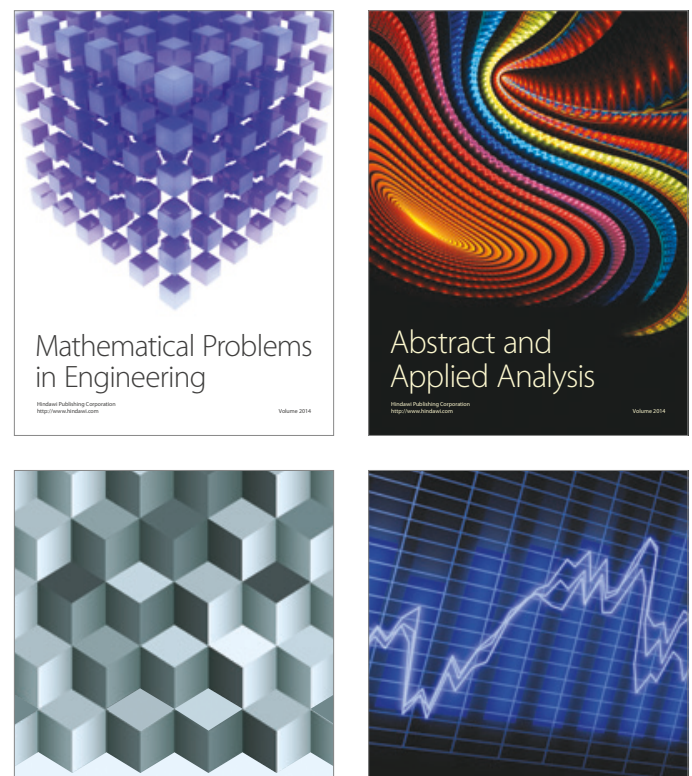

Journal of

Function Spaces

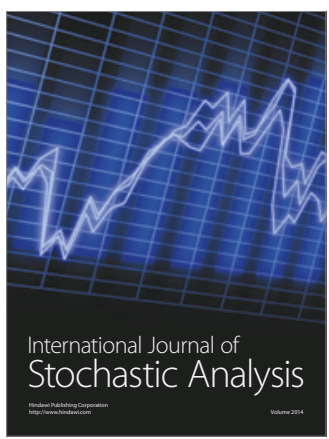

Probability and Statistics
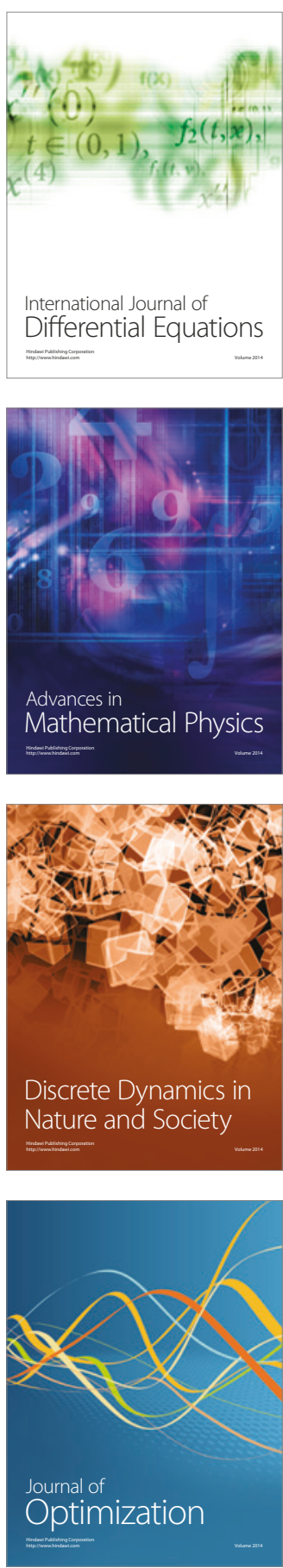